\title{
Procedimientos Utilizados por Estudiantes de 13-14 Años en la Resolución de Tareas que Involucran el Área de Figuras Planas
}

\author{
Procedures Used by Students Aged 13-14 in the Resolution of Tasks \\ Involving the Flat-Figure Area
}

\author{
Sofía Caviedes* \\ ORCID iD 0000-0002-5304-212X \\ Genaro de Gamboa** \\ ORCID iD 0000-0003-0366-3988 \\ Edelmira Badillo*** \\ ORCID iD 0000-0001-6296-4591
}

\begin{abstract}
Resumen
Este estudio pretende identificar la puesta en práctica de diferentes significados parciales del área en estudiantes de 13-14 años cuando resuelven tareas de área. Se analizan procedimientos y justificaciones escritas en tres tareas y se identifican los significados parciales del área utilizados. Se establecen seis perfiles de estudiantes, atendiendo a los conceptos, procedimientos, propiedades y representaciones que los estudiantes usan en sus resoluciones. Los resultados muestran una tendencia generalizada de los estudiantes a asociar el área con el uso de fórmulas, evidenciando procedimientos poco variados para medir áreas y escasas relaciones entre los significados parciales del área.
\end{abstract}

Palabras clave: Significados parciales del área. Representaciones semióticas. Medida del área.

\begin{abstract}
This study aims to identify the implementation of different partial meanings of the area in students aged 13-14 when solving area tasks. Written procedures and justifications are analyzed in three tasks and the partial meanings of the area used are identified. Six student profiles are established, according to the concepts, procedures, properties, and representations used by the students. The results show a generalized tendency of students to associate area with the use of formulas, showing little variation in procedures for area measurement and little relationship between the partial meanings of area.
\end{abstract}

Keywords: Area partial meaning. Semiotics representation. Area measurement.

\footnotetext{
* Estudiante del Programa de Doctorado en Educación de la Universidad Autónoma de Barcelona (UAB), Barcelona, España. Dirección postal: Edificio G5, 08193, Bellaterra, Barcelona, España. E-mail: sofia.caviedes@e-campus.uab.cat.

** Doctor en Didáctica de las matemáticas por la Universidad Autónoma de Barcelona (UAB). Profesor en la Facultad de Educación de la Universidad Autónoma de Barcelona (UAB), Barcelona, España. Dirección postal: Campus Bellaterra, Edificio G5, 08193, Bellaterra, Barcelona, España. E-mail: genaro.degamboa@uab.cat.

*** Doctora en Didáctica de las matemáticas por la Universidad Autónoma de Barcelona (UAB). Profesora en la Facultad de Educación de la Universidad Autónoma de Barcelona (UAB), Barcelona, España. Dirección postal: Edificio G5, 08193, Bellaterra, Barcelona, España. E-mail: edelmira.badillo@uab.cat.
} 


\section{Introducción}

Los conceptos vinculados a los procesos de medición son importantes en las matemáticas escolares, pues además de relacionarse de manera directa con problemas extramatemáticos, pueden proporcionar aplicaciones de distintos contenidos aritméticos y pueden ofrecer una fuente común de ilustraciones en álgebra y geometría (HIRSTEIN, 1981). En el caso particular del área, ésta puede servir como un modelo de base para introducir otros conceptos matemáticos, por ejemplo, las fracciones, la multiplicación, el teorema de Pitágoras, además de jugar un papel fundamental en la justificación geométrica de fórmulas algebraicas, como es el caso de las identidades notables.

La fenomenología didáctica de Freudenthal (1983) distingue tres fenómenos que se ven involucrados en la enseñanza del área, el reparto justo, la comparación y reproducción de formas, y la medición. La interrelación entre éstos fenómenos constituye un significado global del área, cuya complejidad puede ser caracterizada mediante el análisis de las configuraciones de objetos primarios de cada fenómeno (PINO-FAN; GODINO; FONT, 2011) a fin de reconstruir el significado global del área como objeto matemático. Así, la complejidad matemática del objeto área puede caracterizarse desde la configuración de problemas, representaciones, definiciones, proposiciones y propiedades del objeto área; procedimientos y técnicas que se aplican al objeto área; y, argumentos sobre el objeto área.

Cada una de estas configuraciones permite identificar significados parciales del área (GODINO; BATANERO; FONT, 2007) y caracterizar, de manera explícita, los objetos primarios que los componen. Las diferentes configuraciones de objetos primarios se elaboran con base en las propuestas de Freudenthal (1983) y Sarama y Clements (2009). En este contexto, el objetivo del presente estudio es caracterizar el uso que hacen estudiantes de 13 14 años de diferentes significados parciales del área cuando resuelven tareas que involucran el área de figuras planas. Para ello, nos centramos en el análisis de los procedimientos, ya que permiten buscar evidencias del uso de cada uno de los significados parciales.

\section{Marco Teórico}

\subsection{Medición del área}

Diversos estudios (D'AMORE; FANDIÑO, 2007; HUANG; WITZ, 2013; KOSPENTARIS; SPYROU; LAPPAS, 2011; ZACHAROS, 2006) han mostrado que un gran 
número de estudiantes, tanto de educación primaria como de secundaria y bachillerato, poseen una comprensión limitada del área, vinculándola únicamente con el uso de cálculos que aplican de forma memorística. Esto puede ser porque los estudiantes no comprenden el significado de las fórmulas, ni cómo éstas se originan (D’AMORE; FANDIÑO, 2007).

Freudenthal (1983) realiza un estudio teórico sobre el área, señalando que ésta es una magnitud que sirve para medir una gran variedad de objetos. En su análisis didáctico fenomenológico del área, el autor recalca la adquisición del área como objeto mental y enfatiza en la dificultad, incluso en cursos avanzados, para adquirir el concepto de área. Así, identifica tres fenómenos, con sus respectivas manipulaciones, que se ven involucrados en el aprendizaje del área como objeto mental: (1) reparto equitativo, situaciones en las que dado un objeto hay que repartirlo; (2) comparación y reproducción de formas, situaciones en las que hay que comparar dos superficies y donde se requiere obtener un reproducción de una superficie con una forma diferente a la inicial; (3) medición, situaciones en las que una superficie aparece ligada a un proceso de medida, ya sea repartir, medir o valorar. Freudenthal (1983) considera que todas estas aproximaciones fenomenológicas son didácticamente aceptables, pero con distinto peso, por lo que es posible elegir una en función del propósito de la actividad.

Desde una perspectiva empírica Sarama y Clements (2009) advierten que la medición del área implica el aprendizaje y la coordinación de ideas variadas. Además, señalan que el proceso de comprender y ejecutar con precisión los procesos de medición del área requiere de la comprensión previa de ciertos conceptos, procedimientos y propiedades fundamentales.

Entre dichos conceptos encontramos el área como atributo, relacionado con asignar un significado numérico a una cantidad de superficie, y las unidades de medida, que deben ser fácilmente reproducibles y divisibles, sin dejar huecos al momento de recubrir la superficie. Los procedimientos corresponden a la partición equitativa, que requiere cortar el espacio bidimensional, física o mentalmente, en partes de igual área y usualmente congruentes; la iteración de unidades, que requiere cubrir una determinada superficie con una unidad bidimensional sin huecos y sin solapamientos; la estructuración espacial, que requiere cubrir una superficie con cuadrados alineados en filas y columnas. Finalmente, las propiedades corresponden a la acumulación y aditividad, que implica componer y recomponer figuras en superficies con áreas equivalentes; la conservación, que implica cortar una superficie y reorganizar sus partes, advirtiendo la inmutabilidad del área; la transitividad, que implica la comparación del área de dos superficies, tomando como referencia el área de una tercera superficie. 


\subsection{Objetos matemáticos, significados y configuraciones}

Desde el enfoque Ontosemiótico del conocimiento y la instrucción matemática, EOS, toda actuación o expresión (verbal, gráfica etc.) realizada por alguien para resolver problemas matemáticos, comunicar a otros la solución obtenida, validarla o generalizarla a otros contextos y problemas, se considera práctica matemática (GODINO; BATANERO, 1998). Así, el significado de los objetos matemáticos se define como el sistema de prácticas realizada por una persona, o compartidas en el seno de una institución, ante determinadas situaciones problemas (GODINO; BATANERO; FONT, 2007, 2019).

En los objetos matemáticos es posible distinguir entre un sentido y un significado. El sentido corresponde al significado parcial del objeto; es decir, el significado de un objeto se puede parcelar en distintos tipos de prácticas más específicas que pueden ser utilizadas en un determinado contexto. De esta manera, el significado parcial corresponde a un subsistema de prácticas (GODINO; BATANERO; FONT, 2007). El significado global del objeto se reconstruye mediante la exploración sistemática de los contextos de uso del objeto y los sistemas de prácticas que intervienen (GODINO; BATANERO; FONT, 2019).

Para definir los significados parciales del área, que aparecen en las matemáticas escolares hasta los 13-14 años de los estudiantes, e identificar los subsistemas de prácticas que los determinan, consideramos los fenómenos propuestos por Freudenthal (1983) y los conceptos, procedimientos y propiedades propuestos por Sarama y Clementes (2009). La conjunción de estos dos enfoques da como resultado diferentes configuraciones de objetos primarios (GODINO; BATANERO; FONT, 2007) que permiten identificar, para cada significado parcial del área, seis tipos de objetos primarios: (1) lenguajes - términos, expresiones, notaciones, gráficos - en sus diversos registros; (2) situaciones problemas aplicaciones intra o extra-matemáticas -, ejercicios; (3) conceptos - introducidos mediante definiciones o descripciones - como recta, punto, número, media, función; (4) propiedades y proposiciones; (5) procedimientos - algoritmos, operaciones, técnicas de cálculo; (6) argumentos - enunciados usados para validar o explicar las proposiciones y procedimientos deductivos o de otro tipo.

\subsection{Registros de representación}

Duval (1995) distingue entre dos clases de transformaciones de representaciones semióticas, que a menudo pueden estar entrelazadas en el mismo proceso matemático de 
resolución: la conversión y el tratamiento. Mientras que las conversiones se dan entre registros diferentes, los tratamientos se producen dentro de un mismo registro. En este sentido, la conversión implica un mayor salto cognitivo, pues requiere que los estudiantes se apropien de representaciones heterogéneas. La comprensión y coordinación de representaciones heterogéneas es lo que Duval (1999) denomina comprensión integrativa. El proceso matemático implica alguna transformación de representación, por lo que un aspecto que adquiere especial relevancia es la relación signo-objeto. Aunque puedan emplearse diversos sistemas de representación, es posible elegir uno en función del propósito de la actividad. En este sentido, la actividad matemática precisa de una coordinación interna que debe ser construida entre los diferentes registros que se pueden elegir y utilizar (DUVAL, 2006).

La naturaleza del área implica la coordinación de representaciones geométricas y numéricas. Sin embargo, es común que en los procesos de enseñanza y aprendizaje el acceso a un objeto matemático se realice vía una de sus representaciones, por ejemplo, la fórmula de base por altura para el área, generando dificultades si no se toma conciencia o no se hace explícita la diferencia entre el objeto y la representación mediante la cual se accede a él, pues es posible que el objeto sea confundido con una representación específica de éste. Es este sentido, una comprensión profunda del área implica la capacidad para coordinar representaciones geométricas, numéricas y algebraicas.

\section{Método}

El trabajo se sitúa en un paradigma interpretativo con un enfoque cualitativo. Mediante el análisis e interpretación de respuestas de estudiantes a tareas de área de figuras planas, se busca identificar perfiles de estudiantes atendiendo a la manera en que éstos utilizan los diferentes significados parciales del área. Para ello, se considera la configuración de objetos del EOS y los registros de representación semiótica. Se realiza un análisis estructurado en dos fases y, en cada fase, se detallan dos etapas. En la primera fase, primera etapa, se realiza un análisis bibliográfico del área (FREUDENTHAL, 1983; SARAMA; CLEMENTS, 2009) e inspirados en el trabajo de Corberán (1996) identificamos cuatro significados parciales del área (SP) que son caracterizados a partir de la configuración de los seis objetos primarios del EOS. Dichos SP son usados como categorías de análisis en la segunda fase. En la segunda etapa, se diseña un cuestionario de siete tareas que permite buscar evidencias del uso de los diferentes SP del área. Estas siete tareas permiten el uso de los diferentes SP del área de manera ascendente. 
En la segunda fase se realiza un análisis de contenido (KRIPPENDORP, 2004) estructurado en dos etapas. En una primera etapa se analizan respuestas de estudiantes considerando la configuración de objetos de cada SP del área. Se buscan evidencias de procedimientos, representaciones, propiedades y conceptos. En una segunda etapa se identifican los perfiles de estudiantes. Para esto, se toman en consideración los SP del área más utilizados y las transformaciones de tratamiento y conversión (DUVAL, 1995).

El periodo de recogida de datos se realizó en el tercer trimestre del curso escolar 20182019. Los participantes fueron 83 estudiantes, con edades comprendidas entre los 13-14 años, de un centro concertado de Educación Primaria y Secundaria de la provincia de Barcelona (España). Los cuestionarios fueron respondidos por los estudiantes de manera individual en una única sesión de 90 minutos y se les permitió el uso de instrumentos de medición. Los estudiantes, dentro de su programa de estudios, habían recibido instrucción previa sobre la medición de áreas de figuras planas.

\subsection{Análisis}

\subsubsection{Primera fase de análisis: una aproximación al área de figuras planas}

En una primera etapa, la complejidad de los objetos matemáticos propuesta por el EOS permite organizar la revisión bibliográfica (CORBERÁN, 1996; FREUDENTHAL, 1983; SARAMA; CLEMENTS, 2009) en subsistemas de prácticas de los que emergen, a modo de categorías, SP del área que se caracterizan de manera explícita mediante la configuración de objetos primarios. Se identifican cuatro SP del área, que van desde un enfoque geométrico, es decir, manipulación de objetos concretos mediante transformaciones rígidas, hacia un enfoque numérico y algebraico. En el Cuadro 1 se presentan, de manera sintetizada, los cuatro SP del área. Se hace énfasis en los procedimientos, ya que permiten buscar evidencias de propiedades, representaciones y conceptos de los diferentes SP, además de transformaciones de tratamiento y conversión.

\begin{tabular}{|l|l|l|}
\hline $\begin{array}{l}\text { Significados } \\
\text { parciales del área }\end{array}$ & Procedimientos asociados a cada SP & $\begin{array}{l}\text { Otros objetos } \\
\text { matemáticos asociados }\end{array}$ \\
\hline $\begin{array}{l}\text { SP1: Porción de } \\
\text { espacio cerrado }\end{array}$ & $\begin{array}{l}\text { Comparan dos o más superficies por superposición } \\
\text { total y/o parcial }\end{array}$ & $\begin{array}{l}\text {-Propiedades: } \\
\text { conservación } \\
\text { acumulación y aditividad } \\
\text { transitividad }\end{array}$ \\
\cline { 2 - 2 } & $\begin{array}{l}\text { Comparan dos o más superficies por recorte yado } \\
\text { pegado }\end{array}$ & $\begin{array}{l}\text {-Representaciones: gráfica } \\
\text {-Conceptos: cantidad de } \\
\text { espacio ocupado } \\
\text { inclusión entre dos o más superficies }\end{array}$ \\
\hline
\end{tabular}




\begin{tabular}{|c|c|c|}
\hline \multirow[t]{3}{*}{$\begin{array}{l}\mathrm{SP}_{2}: \\
\text { Magnitud/atributo }\end{array}$} & $\begin{array}{l}\text { Comparan superficies de manera directa y/o } \\
\text { indirecta identificando que superficies diferentes } \\
\text { en forma pueden tener igual cantidad de superficie }\end{array}$ & \multirow{3}{*}{$\begin{array}{l}\text {-Propiedades: } \\
\text { conservación. } \\
\text { acumulación y aditividad } \\
\text { transitividad } \\
\text {-Representaciones: } \\
\text { gráfica; } \\
\text { simbólica(numérica) } \\
\text {-Conceptos: cantidad de } \\
\text { espacio ocupado; magnitud } \\
\text { que mide a una superficie. }\end{array}$} \\
\hline & $\begin{array}{l}\text { Descomponen de forma conveniente, gráfica o } \\
\text { mentalmente, dos o más superficies (en cuadrados } \\
\text { y/o triángulos) para realizar comparaciones entre } \\
\text { ellas }\end{array}$ & \\
\hline & $\begin{array}{l}\text { Utilizan la reconfiguración por complementariedad } \\
\text { de formas para realizar comparaciones entre las } \\
\text { superficies }\end{array}$ & \\
\hline \multirow{5}{*}{$\begin{array}{l}\mathrm{SP}_{3} \text { : cantidad de } \\
\text { unidades } \\
\text { bidimensionales } \\
\text { que cubren una } \\
\text { superficie }\end{array}$} & $\begin{array}{l}\text { Descomponen superficies en unidades congruentes } \\
\text { y las unidades en subunidades para facilitar la } \\
\text { medida del área de una superficie }\end{array}$ & \multirow{5}{*}{$\begin{array}{l}\text {-Propiedades: } \\
\text { transitividad } \\
\text {-Representaciones: } \\
\text { gráfica; simbólica } \\
\text { (numérica) } \\
\text {-Conceptos: unidades de } \\
\text { medida bidimensionales; } \\
\text { estructuración espacial }\end{array}$} \\
\hline & $\begin{array}{l}\text { Iteran unidades de medida no estándar para } \\
\text { comparar y medir superficies }\end{array}$ & \\
\hline & $\begin{array}{l}\text { Comparan áreas de más de dos superficies } \\
\text { utilizando como referencia el área de una de las } \\
\text { superficies medidas }\end{array}$ & \\
\hline & $\begin{array}{l}\text { Estructuran arreglos bidimensionales en filas y } \\
\text { columnas considerando medidas de longitud. }\end{array}$ & \\
\hline & $\begin{array}{l}\text { Miden áreas como proceso aditivo, contando } \\
\text { unidades y/o subunidades que recubren el espacio } \\
\text { bidimensional }\end{array}$ & \\
\hline \multirow{2}{*}{$\begin{array}{l}\text { SP4: producto de } \\
\text { dos dimensiones } \\
\text { lineales }\end{array}$} & $\begin{array}{l}\text { Utilizan fórmula y calculan áreas dadas las } \\
\text { medidas de longitud }\end{array}$ & \multirow{2}{*}{$\begin{array}{l}\text {-Propiedades: } \\
\text { transitividad } \\
\text {-Representaciones: } \\
\text { simbólica (numérica, } \\
\text { algebraica); gráfica } \\
\text {-Conceptos: longitud, de medida } \\
\text { unidades de ándar; fórmula del área } \\
\text { están }\end{array}$} \\
\hline & $\begin{array}{l}\text { Calculan áreas de rectángulos, triángulos y } \\
\text { cuadrados, identificando la relación entre sus } \\
\text { fórmulas }\end{array}$ & \\
\hline
\end{tabular}

Cuadro 1 - Significados parciales del área

Fuente: elaborado por los autores

En una segunda etapa, los cuatro SP permiten direccionar el diseño de un cuestionario de siete tareas de comparación y medición de áreas. El cuestionario se estructuró de la siguiente manera: dos tareas que admitían únicamente una aproximación geométrica del área (Tareas 1 y 2). En ambas tareas sólo es posible hacer uso de los SP1 o SP2. Cinco tareas que admiten tanto una aproximación geométrica, como aritmética del área (Tareas 3, 4, 5, 6 y 7). En estas tareas es posible hacer uso de dos o más SP del área. Las tareas se ordenan aumentando el número de SP del área, que pueden ser utilizados para resolverlas de manera competente. Así, en la Tarea 7 es posible poner en práctica todos los SP del área. Esto se promueve pidiendo, de manera explícita, el uso diferentes tipos de resoluciones. Por las restricciones de extensión, en este artículo se presentan los diferentes perfiles de estudiantes mostrando, únicamente, evidencias de las resoluciones de los estudiantes a las tareas 2, 3 y 7 del cuestionario (Cuadro 2).

La Tarea 2 permite buscar evidencias de la capacidad de los estudiantes para resolver tareas que admiten aproximaciones geométricas del área. Las tareas 3 y 7 permiten buscar evidencias de la capacidad de los estudiantes para resolver tareas que admiten aproximaciones 
geométricas, numéricas y/o algebraicas del área, ya que, permiten el uso de diferentes SP del área. De esta manera, la consideración de estas tres tareas permite mostrar evidencias de los SP del área que ponen en práctica los estudiantes a lo largo del cuestionario y, permite mostrar evidencias de transformaciones de tratamiento y conversión.

\begin{tabular}{l}
\hline Enunciado \\
TAREA 2: Tomando como referencia el área \\
sombreada de la Figura (T) ¿Cuál o cuáles de las \\
siguientes figuras tienen la misma cantidad de área \\
sombreada? ¿Por qué?
\end{tabular}

Cuadro 2 - Selección de tareas matemáticas presentadas a los estudiantes Fuente: elaborado por los autores

\subsubsection{Segunda fase de análisis: perfiles de estudiantes}

Se realiza un análisis de contenido para identificar los tipos de procedimientos utilizados por los estudiantes. En los procedimientos se buscan evidencias de los diferentes SP del área a partir de la identificación de representaciones, propiedades y conceptos utilizados. Se definen dos etapas para esta fase. En la primera etapa se realiza un análisis individual de las respuestas de los estudiantes. Se buscan evidencias de los procedimientos, propiedades, representaciones y conceptos que los estudiantes ponen en práctica al resolver cada tarea. Además, se buscan evidencias de transformaciones de tratamiento y conversión en las resoluciones.

En la segunda etapa, las respuestas de los estudiantes se codifican en correctas, parcialmente correctas y erróneas. Las respuestas erróneas causadas por errores de cálculo, pero 
que mostraban un uso justificado de los procedimientos, conceptos y propiedades asociados a los SP se contabilizaron como correctas. Las resoluciones incompletas que mostraban un uso justificado de todos los procedimientos se consideraron parcialmente correctas. Además, se consideraron parcialmente correctas aquellas resoluciones que, en la Tarea 2 , no consideran que una de las figuras (A, B, C o D) tiene un área sombreada equivalente a la de la Figura $\mathrm{T}$ (modelo). Las resoluciones en blanco fueron consideradas como resoluciones erróneas.

Una vez codificadas las respuestas, se identifican patrones de resolución de los estudiantes a las tareas presentadas. Para ello se consideran los SP del área más utilizados, además de las transformaciones de tratamiento y conversión. La interpretación de las respuestas de los estudiantes permite diferenciar 6 tipos de perfiles de estudiantes.

Perfil 1. Inconsistente: agrupa las resoluciones en blanco y aquellas que carecen de sentido. No hay evidencia de la puesta en práctica, de manera coherente, de los SP del área. Tampoco se encuentran evidencias de tratamientos y conversiones.

Perfil 2. Procedimental: pone en práctica, mayoritariamente, el $\mathrm{SP}_{4}$. Se asocia el área exclusivamente a valores numéricos (excepción Tarea 2). Se evidencia un uso de los procedimientos vinculados a partición equitativa y del concepto de estructuración espacial. Las propiedades implicadas en la medida del área (acumulación y aditividad; y, conservación) y el concepto de unidad de medida no son usados. En general las resoluciones dependen de cálculos para medir áreas, lo que ocasiona dificultades al comparar áreas en contextos geométricos y en figuras no prototípicas (Tarea 1, Figura 1). A continuación, en la Figura 1, se presenta un ejemplo de respuestas de estudiantes ubicados en este perfil.

En la Tarea 3 no se relaciona el área del rectángulo y del triángulo con las unidades cuadradas en las que se ha descompuesto la figura. Existen dificultades para establecer una correcta relación entre las áreas de rectángulos y triángulos (el triángulo tiene la mitad del área del rectángulo de igual base y altura que lo contiene) y, no se relaciona las medidas de longitud con la estructura de filas y columnas del rectángulo. Por otro lado, es posible que se consideren iguales los objetos matemáticos área y longitud al no considerar, por ejemplo, los $\mathrm{cm}^{2}$ como la unidad que mide al área (Figura 1).

Finalmente, no hay evidencia de tratamientos y conversiones, pues el área se ve reducida sólo a una de sus representaciones, la fórmula de base por altura. En este sentido, la capacidad de los estudiantes de resolver las tareas de área se ve limitada por el uso de fórmulas. 


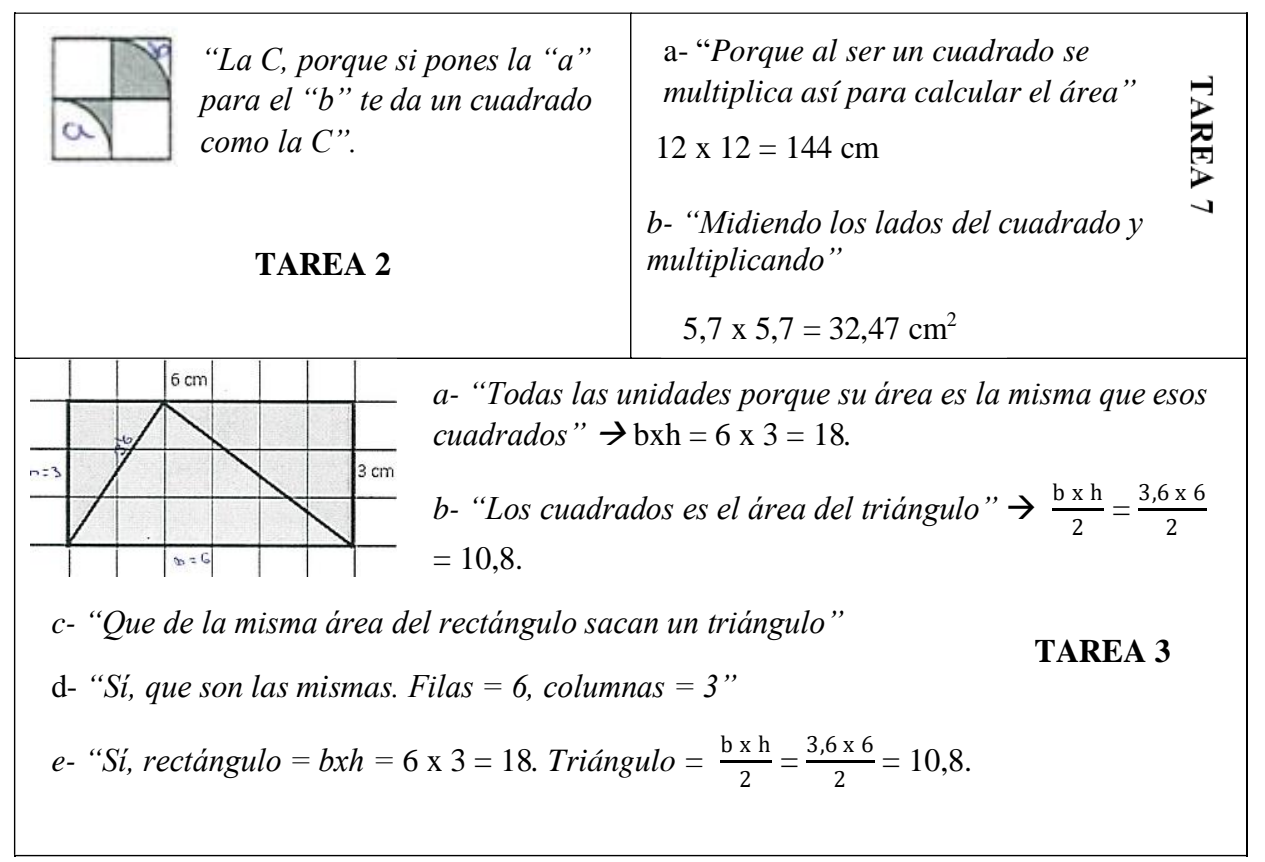

Figura 1 - Ejemplo prototípico de las resoluciones de estudiantes de este perfil Fuente: elaborado por los autores

Perfil 3. Procedimental-estratégico 1: pone en práctica mayoritariamente el $\mathrm{SP}_{4}$. Evidencia un uso incompleto de las propiedades de conservación y acumulación y aditividad, así como, también del concepto de estructuración espacial. Puede utilizar dos SP del área en al menos dos tareas y resolver bien al menos cinco tareas. Se evidencian dificultades al comparar áreas en contextos geométricos y en figuras no prototípicas, tal y como se ilustra en la respuesta a la Tarea 2 (Figura 2). En la Tarea 3 (Figura 2) se relaciona, por influencia de valores numéricos, el área del rectángulo y el triángulo del centro. Sin embargo, existen dificultades para relacionar las medidas de longitud, del rectángulo y del triángulo, con la estructura de filas y columnas del rectángulo.

En la Tarea 7 (Figura 2) se identifica que el lado del cuadrado negro puede ser 1/3 de la diagonal del blanco, pero no se advierte que la diagonal no posee la misma medida que el lado del cuadrado grande, por lo que la respuesta es errónea. Es posible que se consideren iguales los objetos matemáticos área y longitud al no considerar, por ejemplo, los $\mathrm{cm}^{2}$ como unidad que mide al área (Figura 2). No se encuentran evidencias de tratamientos y conversiones, pues se sigue asociando el área con su registro de representación numérico. En este sentido, al igual que en el perfil anterior, la capacidad de los estudiantes de resolver tareas de área se ve limitada por el uso de fórmulas. 


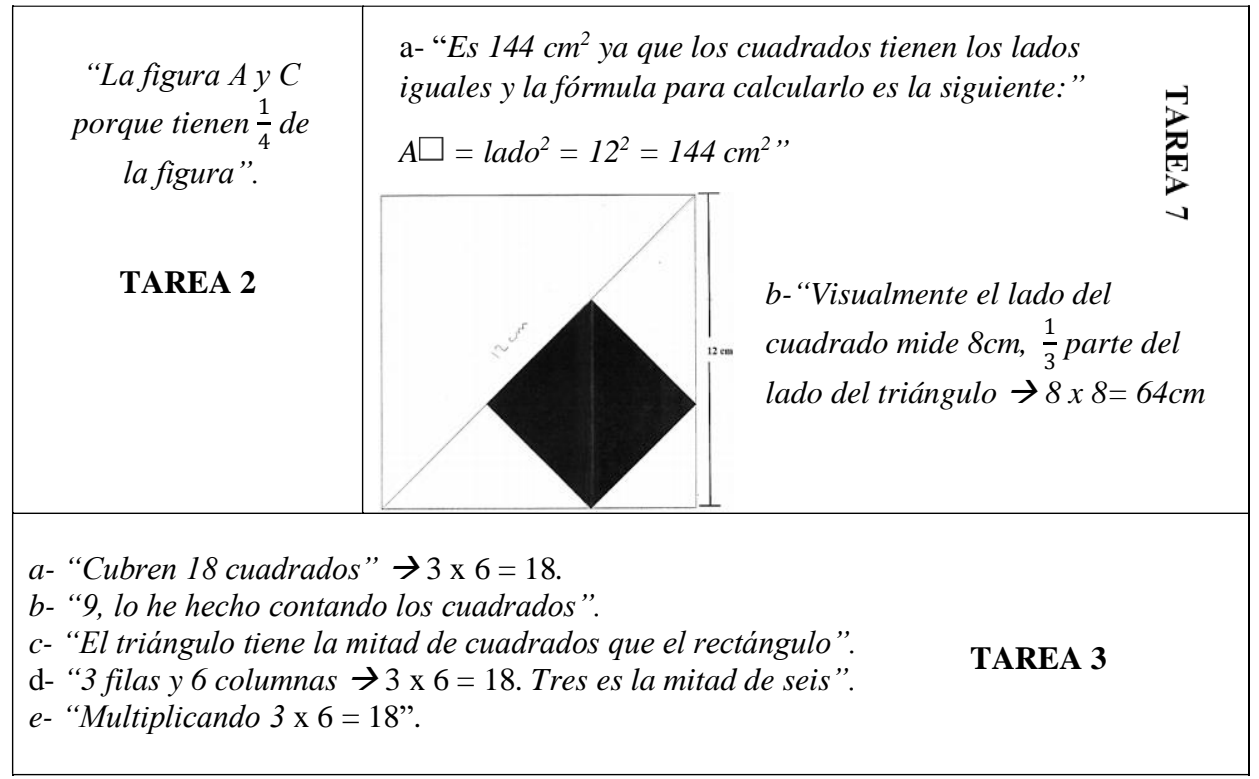

Figura 2 - Ejemplo prototípico de las resoluciones de estudiantes de este perfil Fuente: elaborado por los autores

Perfil 4. Procedimental-estratégico 2: pone en práctica mayoritariamente el $\mathrm{SP}_{4}$. Existe una aproximación tanto del uso de las propiedades de conservación y de acumulación y aditividad, como de los procedimientos vinculados a estructuración espacial y de las unidades de medida. Se relacionan dos SP del área en dos o más tareas y se resuelven correctamente al menos seis tareas. Se evidencian errores producto de la adquisición incompleta de procedimientos, conceptos y propiedades (Figura 3).

Se observa un uso adecuado de los $\mathrm{SP}_{2}$ y $\mathrm{SP}_{4}$. Por ejemplo, en la Tarea 7 (Figura 3) se descompone el cuadrado negro y se obtiene el área por medio de la suma del área de los triángulos que lo componen (acumulación y aditividad). En la Tarea 2 (Figura 3), con base en estimaciones visuales, se concluye que tres de las figuras poseen un área equivalente, pero no se advierte que todas las figuras poseen área equivalente (conservación del área). Por último, en la Tarea 3 (Figura 3) se establece una correcta relación, por influencia de valores numéricos, entre las áreas de rectángulos y triángulos. Se menciona, erróneamente, que cada $\mathrm{cm}$ es una unidad cuadrada, pero no se dice que las medidas de longitud del rectángulo y del triángulo del centro están dadas por la estructura de filas y columnas del rectángulo.

Se utilizan procedimientos más variados, pero se sigue asociando el área con su registro de representación numérico basado en fórmulas. Sin embargo, es posible evidenciar en la Tarea 7 (Figura 3), transformación entre tratamientos dentro de un registro de representación gráfico al descomponer la superficie del cuadrado negro en triángulos congruentes y visualizar la cantidad de triángulos que componen el cuadrado negro. Además, se evidencia conversión entre un registro de representación gráfico y numérico, al descomponer la superficie en triángulos 
congruentes y obtener el área del cuadrado negro por medio de multiplicar el área del triángulo por cuatro. En este sentido, la capacidad de resolver tareas de área podría considerarse mayor que los perfiles anteriores.

\begin{tabular}{|c|c|c|}
\hline $\begin{array}{l}\text { "La figura A, B } \\
\text { y C porque son } \\
\text { el área de un } \\
\text { cuadrado, pero } \\
\text { la D no cumple } \\
\text { la regla". }\end{array}$ & $\begin{array}{l}a-" \text { "El área del cuadrado de } 12 \\
\text { cm }^{2}, \text { ya que todos sus lados son } \\
\text { superficie se han de multiplicar } \\
\mathrm{A}=\mathrm{C}^{2} \\
\mathrm{~A}=12^{2} \\
\mathrm{~A}^{2}=144 \mathrm{~cm}^{2} \\
b \text { - "Puedes medir con una } \\
\text { regla una de sus lados y poner } \\
\text { en práctica su fórmula" } \\
\mathrm{A}=\mathrm{C}^{2} \\
\mathrm{~A}=5,5^{2} \\
\mathrm{~A}=30,25 \mathrm{~cm}^{2}\end{array}$ & $\begin{array}{l}\text { m de lado tiene un área de } 144 \\
\text { guales y para calcular la } \\
\text { los de sus lados" } \\
\text { TAREA } 7 \\
\text { "Puedes dividir el cuadrado } \\
\text { en dos y calcular el área de } \\
\text { los dos triángulos y luego } \\
\text { sumarlo" } \rightarrow \mathrm{A}=\frac{b \times h}{2} \rightarrow \\
\mathrm{A}=\frac{8 \times 4}{2} \rightarrow \mathrm{A}=16 \mathrm{~cm}^{2} \\
\mathrm{~A}=16 \times 2=32 \mathrm{~cm}^{2}\end{array}$ \\
\hline $\begin{array}{l}\text { a- "18 porque el ár } \\
\text { b- "9, porque el áre } \\
\text { c- "Que el triánguld } \\
\text { d- "3 filas y } 6 \text { colun } \\
\text { e- "Multiplicando lc }\end{array}$ & $\begin{array}{l}\text { l rectángulo es base por altura". } \\
\text { triángulo es base por altura entr } \\
\text { a mitad del rectángulo". } \\
\text { Cada cm es una unidad cuadrad } \\
\text { e por la altura } \rightarrow 3 \times 6=18 \mathrm{~cm}^{2}\end{array}$ & dos". \\
\hline
\end{tabular}

Figura 3 - Ejemplo prototípico de las resoluciones de estudiantes de este perfil Fuente: elaborado por los autores

Perfil 5. Estratégico: pone en práctica 2 o 3 SP del área. Depende de cálculos para medir áreas, pero complementa con el uso de procedimientos geométricos. Evidencia más complejidad en el objeto matemático de área, mostrando un uso más estratégico de algunos conceptos, procedimientos y propiedades implicados en la medida del área (unidad de medida, estructuración espacial, acumulación y aditividad, conservación del área y transitividad).

En la Tarea 2 (Figura 4), se identifica que todas las figuras tienen un área equivalente, estableciendo relaciones correctas con base en estimaciones visuales, descomposiciones convenientes de superficies y movimientos de traslación y/o rotación. En la Tarea 7 (Figura 4) se evidencia una correcta relación entre las figuras en las que se ha descompuesto el cuadrado grande y el cuadrado negro. Se calcula el área de cada uno de los triángulos que rodean el cuadrado negro y luego se resta al área total del cuadrado grande (acumulación y aditividad). En la Tarea 3 se evidencia un uso estratégico de $\operatorname{los} \mathrm{SP}_{2}, \mathrm{SP}_{3}$ y $\mathrm{SP}_{4}$. Se identifica, con ayuda de cálculos, que el triángulo del centro y los dos de cada esquina al interior del rectángulo corresponden a 1/2 del área total del rectángulo. Así, se establece una relación correcta entre el área de rectángulos y triángulos y se aproxima a que las medidas de longitud están dadas por la estructura de filas y columnas. Además, se obtiene el área del rectángulo por medio de la suma de las áreas de los tres triángulos que lo componen (Figura 4), evidenciando conversión entre 
un registro gráfico, al visualizar cuántos triángulos componen el rectángulo, y, un registro numérico al obtener el área del rectángulo por medio de la suma de las áreas de los triángulos.

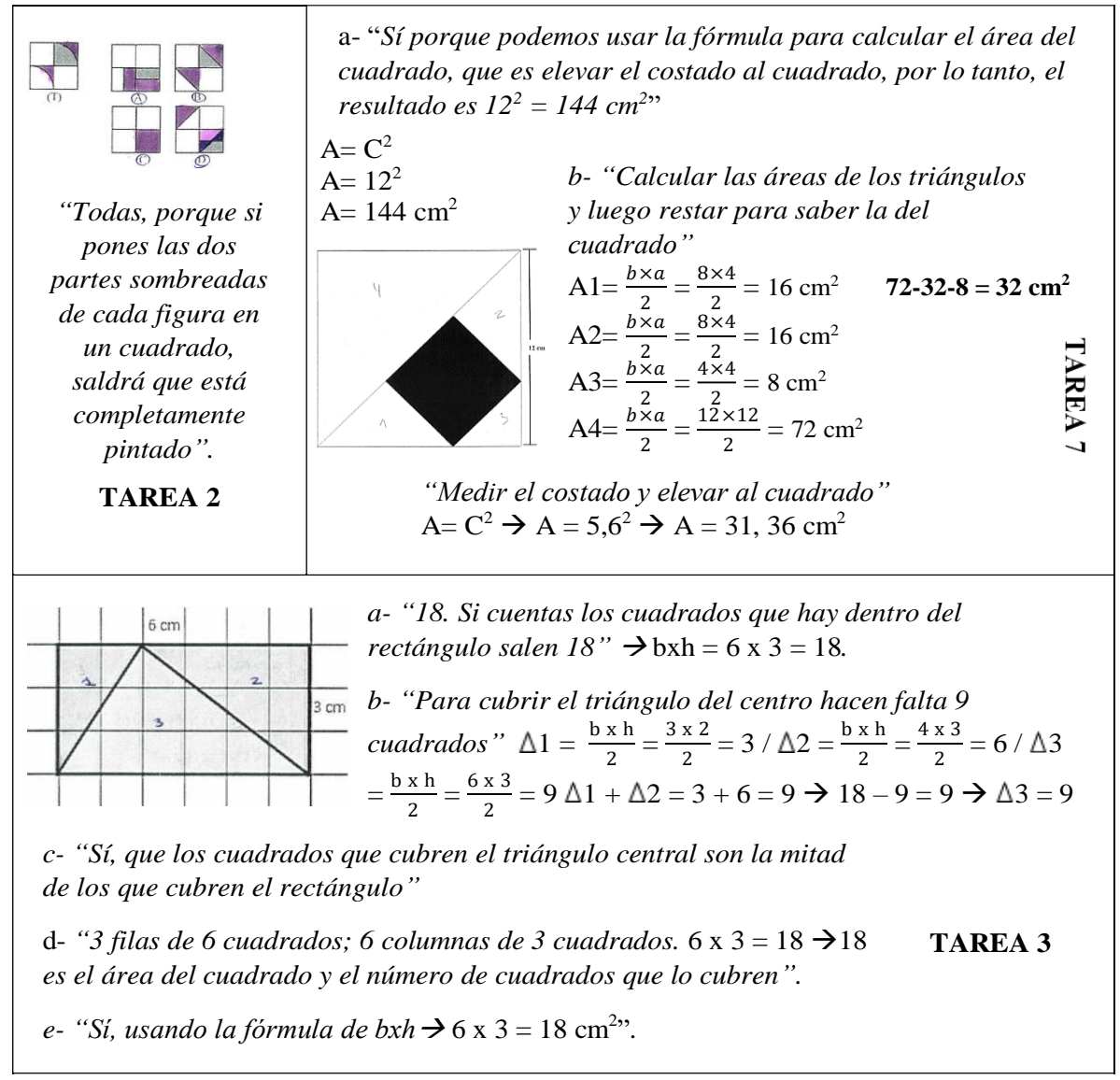

Figura 4 - Ejemplo prototípico de las resoluciones de estudiantes de este perfil Fuente: elaborado por los autores

En la Tarea 2 se evidencia, dentro de un registro de representación gráfico, transformaciones de tratamiento. Se descompone la figura D (Tarea 2, Figura 4) de forma conveniente y luego, por medio del procedimiento de reconfiguración por complementariedad de formas, se visualiza que el área representada es $1 / 4$ del cuadrado. Para las figuras A, B y T (Tarea 2, Figura 4) se realizan movimientos de rotación y/o traslación, para luego visualizar que las áreas son equivalentes. Por último, en la Tarea 7 se observa una conversión entre registro gráfico y numérico. Primero se visualiza la cantidad de triángulos en las que se ha dividido el cuadrado negro y, posteriormente, por medio del uso de fórmulas y operaciones de adición y sustracción, se obtiene el área del cuadrado negro. Además, se observa tratamiento dentro de un registro numérico, pues el área del cuadrado negro se obtiene por medio del uso de fórmula y, por medio de restar al triángulo que conforma la mitad inferior del cuadrado de $12 \mathrm{~cm}$ de lado, las áreas de los triángulos pequeños y medianos. En este sentido, se observa un uso más complejo del objeto matemático de área, mostrando un uso más justificado de los conceptos y propiedades implicados en la medida del área. 
Perfil 6. Estratégico-sofisticado: pone en práctica 2 o 3 SP del área. Se muestran procedimientos más variados pudiendo medir áreas sin llegar a calcularlas. Se evidencia un uso más estratégico y sofisticado del objeto matemático de área, aplicando todos los conceptos y propiedades implicados en la medida del área. En este perfil se ubica sólo a una alumna y en la Figura 5, se presenta su respuesta a las tareas 2, 3 y 7.

En la Tarea 2 se identifica que todas las figuras tienen un área equivalente, estableciendo relaciones correctas entre las áreas sombreadas con base en estimaciones visuales basadas en la propiedad de conservación y concepto de acumulación y aditividad. En la Tarea 3 se evidencia un uso estratégico de los $\mathrm{SP}_{2}, \mathrm{SP}_{3}, \mathrm{SP}_{4}$. Por medio de descomposiciones convenientes del triángulo del centro, se identifica que los triángulos de las esquinas equivalen al triángulo del centro, concluyendo que el área del triángulo solicitado es $1 / 2$ del área total del rectángulo. Se evidencia un uso justificado de la propiedad de transitividad y se establece una relación entre el área de rectángulos y triángulos. Además, se identifica que las medidas de longitud están dadas por la estructura de filas y columnas. En la Tarea 7 se evidencia un uso estratégico de los $\mathrm{SP}_{2}$ y $\mathrm{SP}_{3}$ al descomponer el cuadrado grande en cuadrados y triángulos más pequeños y determinar el área del cuadrado negro por relaciones de inclusión. Esto se complementa con el uso de fórmulas, sin embargo, puede determinar el área prescindiendo de cálculos.

Se observan evidencias de tratamientos y conversiones. En la Tarea 2 se observan transformaciones de tratamiento dentro de un registro de representación gráfico. Se descompone la Figura D (Tarea 2, Figura 5) de forma conveniente y luego, por medio del procedimiento de reconfiguración por complementariedad de formas, se visualiza que el área representada es 1/4 del cuadrado. Para las figuras A, B y T (Tarea 2, Figura 5) se realizan movimientos de rotación y/o traslación, para luego visualizar que las áreas son equivalentes. Para la Tarea 3 se observan transformaciones de tratamiento dentro de un registro de representación gráfico y dentro de un registro de representación numérico. Se descompone el triángulo del centro de forma conveniente y luego, por medio de superposición de las formas en que se ha descompuesto la figura, se visualiza que el triángulo del centro es la mitad del rectángulo (respuesta $b$ Figura 5). En la respuesta $a$ (Tarea 3, Figura 5) obtiene el área del rectángulo por medio del conteo de unidades cuadradas y por medio del producto entre el número de unidades cuadradas de filas y columnas. 


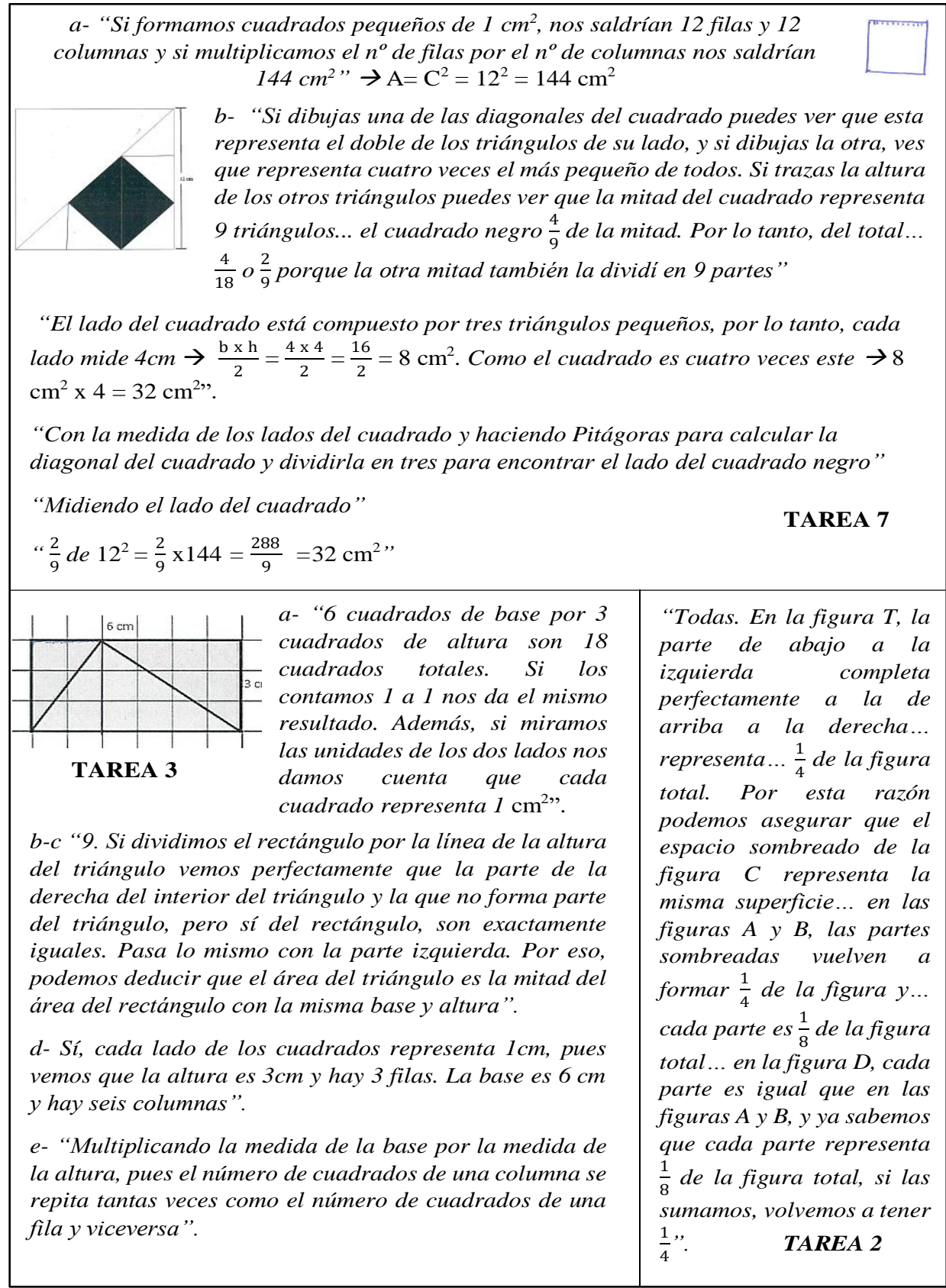

Figura 5 - Único ejemplo de resolución de estudiantes en este perfil Fuente: elaborado por los autores

Finalmente, se observa conversión entre un registro de representación gráfico y numérico en la respuesta $e$ (Tarea 3, Figura 5). Se visualiza que la cantidad de unidades cuadradas en cada columna se repite, tantas veces como, la cantidad de unidades cuadradas en cada fila. Así, se obtiene el área por medio del producto entre la cantidad de unidades cuadradas de filas y columnas. En la Tarea 7 se observan transformaciones de tratamiento y conversión. En la respuesta $a$ (Tarea 7, Figura 5) se observan tratamientos dentro de un registro de representación numérico. Se utiliza la fórmula del área del cuadrado, se multiplica el número de filas por el número de columnas y se cuentan los cuadrados del interior para obtener el área del cuadrado de $12 \mathrm{~cm}$ de lado. 
Se evidencian tratamientos dentro de un registro de representación gráfico, ya que, se descompone la superficie cuadrada en cuadrados de $1 \mathrm{~cm}$ de lado y, luego, se visualiza la cantidad de filas y columnas. Además, se observa conversión entre un registro de representación gráfico y numérico, pues se descompone la superficie cuadrada en cuadrados de $1 \mathrm{~cm}$ de lado y se obtiene el área por medio de un proceso aditivo y, multiplicativo. En la respuesta $b$ (Tarea 7 Figura 5), se observan transformaciones de tratamiento dentro de un registro de representación gráfico. Se descompone la superficie cuadrada en triángulos congruentes y se visualiza la cantidad de triángulos que componen el cuadrado negro.

Dentro de un registro de representación numérico, se cuenta el número de triángulos que componen ambos cuadrados y se establece la proporción que ocupa el cuadrado negro respecto del cuadrado grande en un registro de representación numérico fraccionario. De forma similar, se evidencian tratamientos dentro de un registro de representación gráfico, pues se descompone la superficie del cuadrado negro en triángulos congruentes y se visualiza la cantidad de triángulos que lo componen.

Se observan conversiones entre un registro de representación gráfico y numérico. Se descompone la superficie del cuadrado grande en unidades congruentes y se obtiene el área del cuadrado negro por medio del conteo de tales unidades y por medio del cálculo de la fracción de una cantidad. Además, se evidencia conversión entre un registro de representación gráfico y numérico, pues se descompone la superficie en triángulos congruentes y se obtiene el área del cuadrado negro por medio del producto entre el área de un triángulo y cuatro.

\section{Discusión y Resultados}

Los hallazgos muestran que una mayoría de estudiantes tiene un repertorio limitado de procedimientos para resolver tareas que responden a contextos geométricos. Por el contrario, en contextos numéricos donde la fórmula puede ser aplicada de manera directa responden, en su mayoría, únicamente con uso de cálculos dejando de lado procedimientos de naturaleza geométrica que podrían simplificar la resolución de las tareas. La Tabla 1 muestra que las tareas 3 y 7 presentan el mayor número de resoluciones correctas en aquellos casos donde la fórmula podía utilizarse directamente. Sin embargo, cuando se pregunta por relaciones entre áreas de figuras (3b, 3c y 3d) el número de respuestas correctas disminuye. Así mismo, sucede cuando deben emplear más de un procedimiento para encontrar áreas (7b). 
Tabla 1 - Resumen de respuestas de estudiantes Tareas 1,2 y 3. $(\mathrm{N}=83)$

\begin{tabular}{lllll}
\hline Tarea & Correcto & $\begin{array}{l}\text { Parcialmente } \\
\text { correcto }\end{array}$ & Incorrecto & s/respuesta \\
\hline $\mathbf{2}$ & 11 & 62 & 9 & 1 \\
\hline $\mathbf{3 a}$ & 67 & - & 11 & 5 \\
\hline $\mathbf{3 b}$ & 35 & - & 39 & 9 \\
\hline $\mathbf{3 c}$ & 34 & - & 37 & 12 \\
\hline $\mathbf{3 d}$ & 41 & 2 & 35 & 5 \\
\hline $\mathbf{3 e}$ & 55 & 1 & 16 & 10 \\
\hline $\mathbf{7 a}$ & 59 & 2 & 13 & 9 \\
\hline $\mathbf{7 b}$ & 38 & 5 & 27 & 13 \\
\hline
\end{tabular}

Fuente: elaborado por los autores

Los resultados mostrados en la Tabla 2 evidencian dificultades en el uso de los diferentes SP del área, ya que una minoría de estudiantes se encuentra en los perfiles estratégico y estratégico-sofisticado (siete estudiantes y un estudiante, respectivamente). En este caso, al utilizar dos o más SP del área, los estudiantes utilizan representaciones equivalentes, ya sea por medio de procedimientos de naturaleza geométrica, numérica o ambos a la vez. Así, evidencian un uso justificado de los conceptos, procedimientos y propiedades vinculados a la medida del área. Esta tipología de estudiantes puede razonar desde la estructura multiplicativa (Figuras 4 y 5), midiendo áreas sin necesidad de calcularlas (Figura 5), sino por medio de comparaciones y relaciones entre superficies.

Tabla 2 - Número de estudiantes en cada perfil. $\mathrm{N}=83$

\begin{tabular}{lcc}
\hline & Perfil & $N^{\circ}$ de estudiantes \\
\hline Inconsistente & 23 \\
\hline Procedimental & 4 \\
\hline a- Procedimental-estratégico 1 & 24 \\
b- Procedimental-estratégico 2 & 24 \\
\hline Estratégico & 7 \\
\hline Estratégico-comprensivo & 1 \\
\hline
\end{tabular}

Fuente: elaborado por los autores

La mayoría de estudiantes se ubica en los perfiles procedimental-estratégico 1 y 2 (48 estudiantes), lo que indica una tendencia al uso de fórmulas para medir y comparar áreas. Existe una aproximación hacia la puesta en práctica de los diferentes SP del área (2, 3 y 4), pero con dificultades, como se evidencia en las figuras 3 y 4 . Resulta llamativo el alto número de estudiantes que se ubican en el perfil inconsistente (23), ya que evidencian un uso limitado de conceptos, procedimientos y propiedades involucrados en la medida del área.

En la Figura 6 es posible observar el número de SP del área que ponen en práctica los estudiantes en cada tarea. Los valores ubicados en el centro corresponden al número de SP del área que son utilizados en cada tarea. El valor mínimo es cero, pues el perfil inconsistente no evidencia uso coherente de los SP del área. El valor máximo es 3 debido a que el perfil más avanzado utiliza los $\mathrm{SP}_{2}, \mathrm{SP}_{3}$ y $\mathrm{SP}_{4}$ del área. Esto se debe, principalmente, a que el $\mathrm{SP}_{1}$ considera 
procedimientos con ausencia de razonamiento numérico; es decir, se utilizan expresiones como: más grande, más pequeño, menos, más, entre otros. Pero los estudiantes, debido a su instrucción previa posiblemente, tienen incorporado el razonamiento numérico. El círculo rojo indica la presencia de conversiones y el circulo azul, de tratamientos.

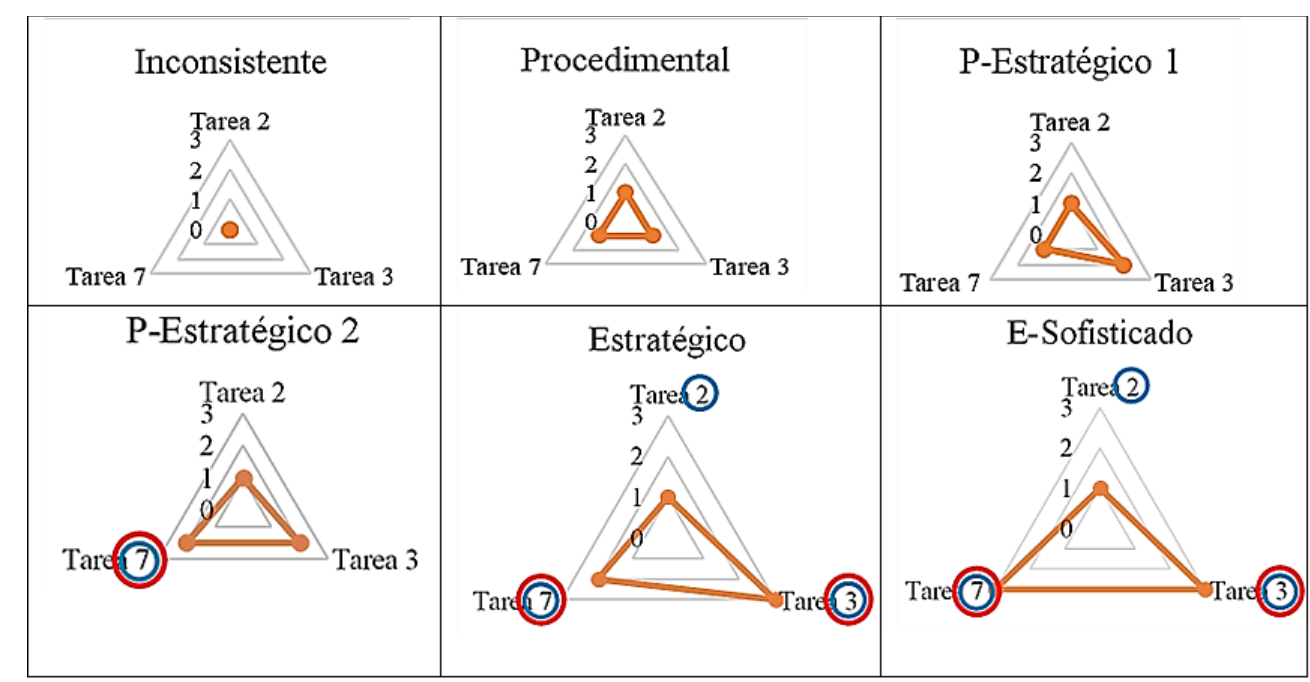

Figura 6 - Número de SP del área que utiliza cada perfil de estudiantes. Fuente: elaborado por los autores

Se puede ver que el perfil procedimental se mueve utilizando mayoritariamente $\mathrm{SP}_{4}$, a excepción de la Tarea 2, donde recurre a estimaciones visuales para establecer relaciones entre áreas, pero de forma incompleta (Figura 1). Por su parte el perfil procedimental-estratégico 1 hace uso de un $\mathrm{SP}$ en las tareas 2 y $3, \mathrm{SP}_{2}$ y $\mathrm{SP}_{4}$, respectivamente y, utiliza $\mathrm{SP}_{3}$ y $\mathrm{SP}_{4}$;, $\mathrm{SP}_{2}$ y $\mathrm{SP}_{4}$ en la Tarea 2, pero con errores (Figura 4). El perfil procedimental-estratégico 2 utiliza el $\mathrm{SP}_{2}$ en la Tarea 2, y utiliza $\mathrm{SP}_{3}$ y $\mathrm{SP}_{4}$;, $\mathrm{SP}_{2}$ y $\mathrm{SP}_{4}$ en las tareas 3 y 7 , con errores y aciertos como se observa en la Figura 3. En este perfil hay conversiones y tratamientos en la Tarea 7.

El perfil estratégico utiliza el $\mathrm{SP}_{2}$ para la Tarea 2; $\mathrm{SP}_{2}, \mathrm{SP}_{3}$ y $\mathrm{SP}_{4}$ para la Tarea 3; y $\mathrm{SP}_{3}$ o $\mathrm{SP}_{2}$ y $\mathrm{SP}_{4}$ en la tarea 7, logrando establecer relaciones entre conceptos, procedimientos, representaciones y propiedades (Figura 4). Hay tratamientos en las tareas 2, 3 у 7 у conversiones en las tareas 3 y 7. Por último, el perfil estratégico-sofisticado utiliza sólo $\mathrm{SP}_{2}$ para la Tarea 2; $\mathrm{SP}_{2}, \mathrm{SP}_{3}$ y $\mathrm{SP}_{4}$ para las tareas 2 y 3 . Este perfil muestra un uso más estratégico y sofisticado del objeto matemático de área, justificando los conceptos, procedimientos y propiedades. Así mismo, muestra evidencias de un mayor número de transformaciones de tratamiento y conversión, en relación a los perfiles anteriores. 


\section{Conclusiones}

Los resultados de este estudio indican que una mayoría de estudiantes manifiestan un uso incompleto de los conceptos, procedimientos y propiedades asociados a la medida del área. Esto se evidencia en el escaso número de casos que son capaces de resolver todas las tareas de manera competente. El uso de los diferentes SP del área se ve restringido por el conocimiento de la fórmula del área de cuadrados y triángulos. De esta manera, se confirman resultados de investigaciones anteriores (D’AMORE; FANDIÑO, 2007; HUANG; WITZ, 2013; KOSPENTARIS; SPYROU; LAPPAS, 2011; ZACHAROS, 2006), que concluyen que el desempeño de los estudiantes queda limitado casi exclusivamente por el uso de cálculos y fórmulas, aun cuando no se precisa de éstos.

La definición de los perfiles de estudiantes permite concluir que el uso de registros de naturaleza geométrica puede vehiculizar la adquisición de la competencia aritmética de una manera comprensiva, entendiendo qué se hace y porqué se hace. De esta manera, hacer transformaciones geométricas llevaría asociado un mejor uso de fórmulas y una capacidad para justificar mejor los procedimientos que se utilizan. La caracterización de los diferentes perfiles muestra una relación entre el uso de diferentes significados parciales y la competencia de los estudiantes para enfrentarse a la resolución de tareas matemáticas que involucran el área de figuras planas. La coordinación estratégica de diferentes SP del área implica el establecimiento de conversiones entre registros geométricos y numéricos, sustentadas por el uso justificado de los conceptos, procedimientos y propiedades propuestos por Sarama y Clements (2009), lo que se asocia, también con justificaciones más elaboradas por parte de los estudiantes.

El enfoque geométrico presentado puede ser útil para el diseño didáctico de tareas de área, tanto a lo largo de la educación obligatoria como en la formación inicial de maestros. Los resultados muestran que es necesario introducir tareas que incentiven el uso de registros de representación de naturaleza geométrica, numérica y algebraica, con el objetivo de favorecer una concepción profunda del área como objeto matemático y el uso estratégico de fórmulas.

\section{Agradecimientos}

Estudio financiado por ANID PFCHA/DOCTORADO BECAS CHILE/201872190032, EDU2015-65378-P, MINECO/FEDER, GIPEAM, SGR-2017-101, AGAUR. Estudio realizado en el Programa de Doctorado en Educación de la Universidad Autónoma de Barcelona, España. 


\section{Referencias}

CORBERÁN, R. Análisis del concepto de área de superficies planas: Estudio de su comprensión por los estudiantes desde primaria a la universidad. Tesis (Doctorado en Ciencias de la Educación) Facultad de Educación, Universidad de Valencia, Valencia, 1996.

D'AMORE, B.; FANDIÑO, M. Relaciones entre área y perímetro: convicciones de maestros y de estudiantes 1. Revista latinoamericana de investigación en matemática educativa, México D.F, v. 10, n. 1, p. 39-68, 2007.

DUVAL, R. Geometrical pictures: Kinds of representation and specific processings. In: SUTTHERLAND, R.; MASON, J. (ed.). Exploiting mental imagery with computers in mathematics education. Berlín: Springer, 1995. p. 142-157.

DUVAL, R. Representation, vision and visualisation: cognitive functions in mathematical thinking. In: HITT, F.; SANTOS, M. (ed.). Proceedings of the $21^{\text {st }}$ Annual Meeting North American PME. Columbus: ERIC/CSMEE Publications, 1999. p. 3-26.

DUVAL, R. A cognitive analysis of problems of comprehension in a learning of mathematics. Educational studies in mathematics, New York, v. 61, n. 1, p. 103-131, 2006.

FREUDENTHAL, H. Measuring by means of Geometry. In: BISHOP, A.J. (ed.). Didactical phenomenology of mathematical structures. Dordrecht: Kluwer, 1983. p. 373-392.

GODINO, J. D.; BATANERO, C. Clarifying the meaning of mathematical objects as a priority area for research in mathematics education. In: SIERPINSKA, A.; KILPATRICK, J. (ed.). Mathematics education as a research domain: A search for identity. Dordrecht: Kluwer, 1998. p. 177-195.

GODINO, J. D.; BATANERO, C.; FONT, V. The onto-semiotic approach to research in mathematics education. ZDM, Berlín, v. 39, n. 1-2, p. 127-135, 2007.

GODINO, J. D.; BATANERO, C.; FONT, V. The Onto-Semiotic Approach: Implications for the Prescriptive Character of Didactics. For the Learning of Mathematics, Vancouver, v. 39, n. 1, p. 3843, 2019.

HIRSTEIN, J. J. The second national assessment in mathematics: Area and volume. The Mathematics Teacher, Reston, v. 74, n. 9, p. 704-708, 1981.

HUANG, H.-M. E.; WITZ, K. G. Children's Conceptions of Area Measurement and Their Strategies for Solving Area Measurement Problems. Journal of Curriculum and Teaching, Ontario, v. 2, n. 1, p. 10-26, 2013.

KOSPENTARIS, G.; SPYROU, P.; LAPPAS, D. Exploring students' strategies in area conservation geometrical tasks. Educational Studies in Mathematics, Heidelberg, v. 77, n. 1, p. 105-127, 2011.

KRIPPENDORP, K. Content Analysis: An Introduction to its Methodology. London: SAGE, 2014. $456 \mathrm{p}$.

PINO-FAN, L.; GODINO, J.D.; FONT, V. Faceta epistémica del conocimiento didáctico-matemático sobre la derivada. Educação Matemática Pesquisa, São Paulo, v. 13, n. 1, p. 141-178, 2011.

SARAMA, J.; CLEMENTS, D. H. Geometric Measurement, Part 2: Area, Volume, and Angle. In SCHOENFELD, A. (ed.). Early childhood mathematics education research: Learning trajectories for young children. New York: Routledge, 2009. p. 293-304. 
ZACHAROS, K. Prevailing educational practices for area measurement and students' failure in measuring areas. The Journal of Mathematical Behavior, New York, v. 25, n. 3, p. 224-239, 2006.

Submetido em 02 de Abril de 2020. Aprovado em 28 de Julho de 2020. 